\title{
Assessing the effect of multibracket appliance treatment on tooth color by using electronic measurement
}

\author{
Anja Ratzmann ${ }^{1 *}$, Christan Schwahn², Anja Treichel ${ }^{3}$, Andreas Faltermeier ${ }^{4}$ and Alexander Welk ${ }^{5}$
}

\begin{abstract}
Background: The purpose of this study was to investigate how tooth color is affected by multibracket appliance (MBA) treatment.

Methods: The color of teeth \#14 to \#24 of 15 patients with MBA was measured on body and gingival tooth segments using the spectrophotometer Shade Inspector ${ }^{\mathrm{TM}}$. Colors of both segments were recorded before start of MBA treatment (baseline $\left.T_{0}\right)$, end of MBA treatment $\left(T_{1} ; 2\right.$ years \pm 0.3$)$, and 3 months after $T_{1}\left(T_{2}\right)$. A $2 D$ color system and a 3D system served as reference systems.

Multilevel models were used to analyze color change within segments and to compare the difference in color change between segments (treatment effect).

Results: $2 \mathrm{D}$ system. Changes within tooth segments from $\mathrm{T}_{0}$ to $\mathrm{T}_{2}$ were at worst 2.0 units ( $\Delta \mathrm{E}$ in the gingival segment), which is less than the threshold of 2.7 units for a clinically meaningful difference. Confidence intervals for the treatment effect indicated no clinically important differences in color change between body and gingival segments.

$3 \mathrm{D}$ system. Changes within tooth segments from $T_{0}$ to $T_{2}$ were at worst 2.3 units ( $\Delta E$ in the body segment), which is less than the threshold of 2.7 units for a clinically meaningful difference. Confidence intervals for the treatment effect indicated no clinically important differences in color change between body and gingival segments.

Thus, MBA treatment did not lead to clinically relevant changes in tooth color.

Conclusion: Within the limitation of this study the MBA treatment can be seen as a safe method with respect to tooth color.
\end{abstract}

\section{Background}

Changes in tooth color may be caused by several factors, for instance, by extrinsic (external) and intrinsic (internal) discolorations, or by aging [1]. Further causes of color changes are dental treatments, including bleaching or restorative therapy [2]. In addition, tooth color can be changed by the acid-etching process used for bonding orthodontic brackets [3]. Formation of white spots and irreversible penetration of resin tags that remain in the enamel as the two main causes have been reported [4-7]. Therefore, multibracket treatment (MBA) may be

\footnotetext{
* Correspondence: anja.ratzmann@uni-greifswald.de

${ }^{1}$ Department of Orthodontics and Department of Dental Propaedeutics/ Community Dentistry, Dental School, University Medicine, Walther-Rathenau Straße 42a, 17475 Greifswald, Germany

Full list of author information is available at the end of the article
}

associated with enamel discoloration due to changes in the enamel by tooth cleaning, enamel conditioning procedures (etching), and the debonding and subsequent polishing processes $[8,9]$.

Association between tooth color changes due to bonding and debonding procedure and multibracket treatment (MBA) is discussed controversial. Some studies $[4,10,11]$ have shown that enamel color variables were significantly affected by bonding and debonding procedures, other investigations [3, 12-14] did not find clinically important influence of this procedures on the enamel discolorations.

The purpose of this in vivo study was to investigate how tooth color is affected by multibracket appliance (MBA) treatment, especially whether: (1) the change in tooth color during MBA treatment is clinically important; (2) the color change differs by bracket (body) and 
non-bracket (gingival) tooth segments; and (3) the change is substantially the same for the conventionally used 2D system and the scientifically favorable 3D system.

\section{Methods}

Subjects and clinical examination procedure

All subjects expecting MBA treatment were regular patients of the orthodontic department and participated on a voluntary basis. All measurements were performed during regular visits. All procedures performed in this study were in accordance with the ethical standards of the institutional research committee Ärztekammer Mecklenburg-Vorpommern (Reg. Nr.III UV 15/08). Informed consent was obtained from the patients and parents before start of the study. Initially, 26 patients were included. The inclusion criteria were good oral hygiene, non-carious and restoration-free permanent teeth, and no white spots. The multibracket appliances had been present in situ for $2.0(\mathrm{SD} \pm 0.3)$ years (individual study period of each patient). The entire period of study data collection lasted from 2005 to 2009. Time points of measurements were start of MBA treatment (baseline $\mathrm{T}_{0}$ ), end of MBA treatment ( 2 years $\mathrm{SD} \pm 0.3-\mathrm{T}_{1}$ ), and 3-month after end of MBA treatment $\left(\mathrm{T}_{2}\right)$ (Fig. 1). The complete clinical procedure was performed by an experienced orthodontist under standardized conditions (color neutral such as same room and light conditions, patient was covered by a drape, tooth surfaces were always saliva-wet) according to the standardized bonding protocol of the orthodontic department. Enamel was etched with 35\% orthoposphoric acid (Scotchbond, 3 M Unitek) for $10 \mathrm{~s}$, rinsed with air-water spray for $20 \mathrm{~s}$ and dried for 10 s. Transbond $\mathrm{XT}^{\mathrm{Tm}}$ Ligth Cure Primer (3 M, Unitek) was used in conjunction with Transbond $\mathrm{XT}^{\mathrm{mix}}$ Ligth Cure Adhesive (3 M Unitek) according to the manufacturer's

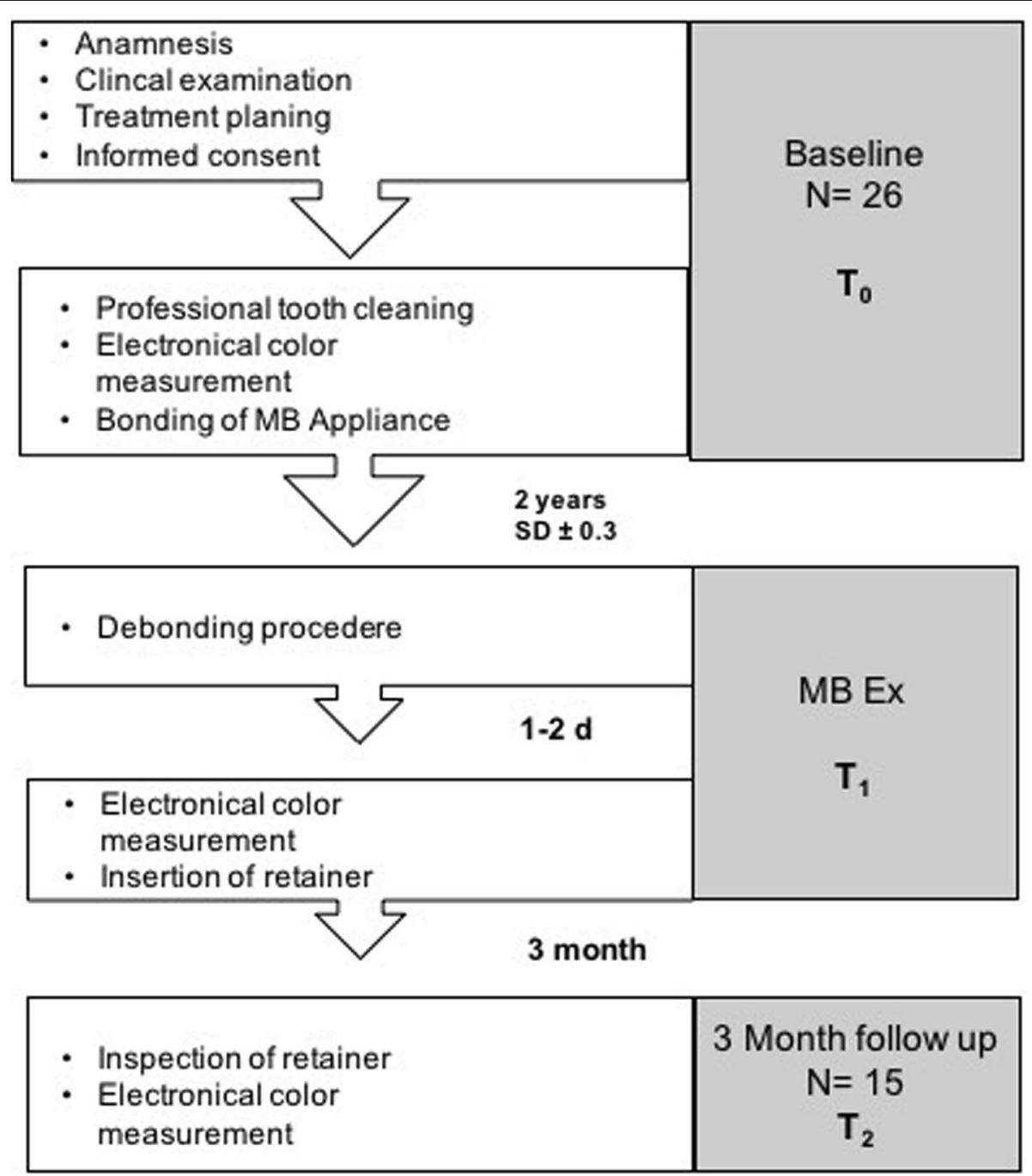

Fig. 1 Consort Flow Diagram 
instructions for bonding Mini-Mono - .022 Roth Technique Stainless Steel Brackets (Forestadent, Germany). After that the bracket was pressed firmly on the enamel surface and the excess adhesive resin was removed with a probe. Light curing was performed with LED source Starlight Pro (Mectron, Germany) for $10 \mathrm{~s}$. For study purposes, the protocol was slightly modified by the additional advice "avoiding etching of the gingival segment". Each tooth was categorized into the gingival $\left(\mathrm{S}_{1}\right)$, the body $\left(\mathrm{S}_{2}\right)$, and the incisal $\left(\mathrm{S}_{3}\right)$ segment (Fig. 2$)$. For standardization of the measurements, we used the facial axis point (FA point) for placing the bracket determined with a Dental Bracket Placement Gauge accordingly the $\mathrm{MBT}^{\mathrm{m}}$-technique for the middle segment $S_{2}$ and for gingival segment $S_{1}$ we placed the tip of the measuring probe perpendicularly $1 \mathrm{~mm}$ above of the middle point of the gingival line of the corresponding tooth (Fig. 3). The probe was moved slightly around the defined measurement points measuring automatically four times giving an overall value of these measurements at the end. The incisal segment $S_{3}$ was not included into analysis because of its transparency. All measurements were performed by a calibrated examiner from a pilot study [15].

During the entire study period we lost 11 patients. Drop out reasons were lack of oral hygiene with breakup of fixed orthodontic treatment, move, repeated schedule failure and withdrawal of informed consent.

\section{Electronic color measurement}

Tooth color was measured electronically with the spectrophotometer Shade Inspector ${ }^{\mathrm{Tm}}$ (Schuetz Dental, Rosbach, Germany- presently not available). The tooth color measuring device operates independently of light on the principle of spectral photometry. For color determination, the color data of the test specimen are compared with manufacturer-furnished color
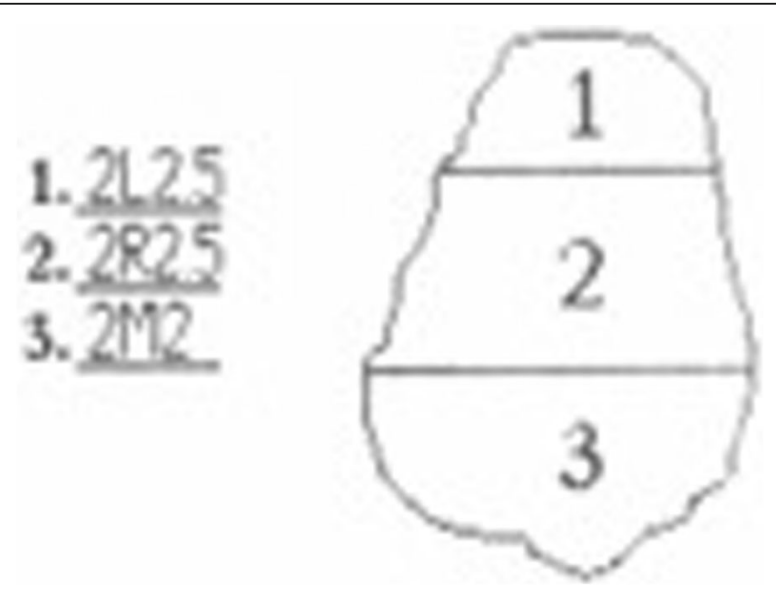

Fig. 2 Measuring report by Shade Inspector ${ }^{\mathrm{TM}}$

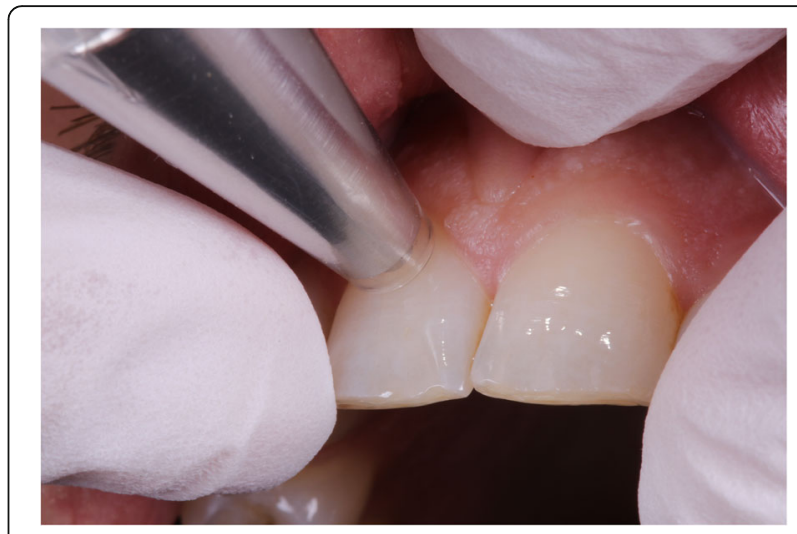

Fig. 3 Shade Inspector ${ }^{\mathrm{TM}}$ - Measurement of the gingival segment $\left(\mathrm{S}_{1}\right)$

rings. The tested spectrophotometer is calibrated with a factory-provided selection of industrially fabricated color reference scale VITAPAN Classical $^{\circ}$ and VITA 3D-Master by the company (Schuetz Dental, Rosbach, Germany). In the present study, the color references VITAPAN Classical ${ }^{\circ}$ and VITA SYSTEM 3D-Master ${ }^{\circ}$ were selected from the device software. The VITAPAN ${ }^{\circ}$ Classical Color System has a two-dimensional structure that enables the description of hue (category A to D) and lightness including chroma (group 1 to 4) [16, 17]. It serves as standard shade guide for visual color assessment in dental praxis. The VITA 3D-Master ${ }^{\circ}$ Color System has a three-dimensional structure that enables the separate description of lightness ( 1 to 5 and 0 for bleaching), chroma ( 1 to 3 , including half points), and hue (M, L, R) [18]. It was developed to obtain a method for systematic and ordered color determination and a better hit rate. The examiners were provided with device operating instructions to ensure observance of the manufacturer's specifications and calibrated in a pilot study [15]. Within a $1 \mathrm{~mm}$ measurement range diameter, the probe measures 26 standard colors and three bleaching colors from the VITA 3D-Master ${ }^{\circ}$ color ring as well as 16 standard colors and 48 intermediate colors (calculated) from the VITA Classical ${ }^{\circ}$ color ring. The measuring probe was protected by a detachable hygiene cap. During the measurements the probe was placed vertically to the tooth surface (Fig. 3).

\section{Statistical methods}

As the 3D-system (VITA 3D-Master) is "a more ordered shade guide" than the 2D-system (VITAPAN ${ }^{\circ}$ Classical) [16], we considered the 3D-system as the primary outcome [19, 20].

Besides lightness and chroma, we analyzed color distributions in terms of $L^{*}$ (CIE lightness) and $C_{a b}^{*}$ (CIE chroma) after having assigned VITA 3D-Master ${ }^{\circ}$ shades to values given in Table 1 in Ahn et al. [21] via data 
Table 1 Description of color distributions on tooth level $(n=120)$

\begin{tabular}{|c|c|c|c|c|c|c|c|c|}
\hline & \multirow{2}{*}{$\begin{array}{l}\text { Time } \\
\text { point }\end{array}$} & \multicolumn{3}{|c|}{ Gingival $\left(S_{1}\right)$} & \multicolumn{3}{|c|}{ Body $\left(S_{2}\right)$} & \multirow{2}{*}{$\begin{array}{l}P \\
\text { value }\end{array}$} \\
\hline & & Mean & Gmd & Median (1st - 3rd quartile) & Mean & Gmd & Median (1st - 3rd quartile) & \\
\hline \multicolumn{9}{|l|}{ 3D system } \\
\hline \multirow[t]{3}{*}{ Lightness $^{a}$} & $\mathrm{~T}_{0}$ & 1.93 & 0.72 & $2(1-2)$ & 1.84 & 0.75 & $2(1-2)$ & 0.170 \\
\hline & $\mathrm{T}_{1}$ & 2.02 & 0.83 & $2(2-2)$ & 1.96 & 0.94 & $2(1-2)$ & \\
\hline & $\mathrm{T}_{2}$ & 2.06 & 0.73 & $2(2-2)$ & 1.98 & 0.73 & $2(2-2)$ & \\
\hline \multirow[t]{3}{*}{ Chroma $^{b}$} & $\mathrm{~T}_{0}$ & 2.45 & 0.40 & $2.5(2.0-2.5)$ & 2.38 & 0.35 & $2.5(2.0-2.5)$ & 0.018 \\
\hline & $\mathrm{T}_{1}$ & 2.48 & 0.39 & $2.5(2.0-2.5)$ & 2.39 & 0.38 & $2.5(2.0-2.5)$ & \\
\hline & $\mathrm{T}_{2}$ & 2.54 & 0.39 & $2.5(2.5-3.0)$ & 2.48 & 0.39 & $2.5(2.0-2.5)$ & \\
\hline \multirow[t]{3}{*}{$L^{*}$} & $\mathrm{~T}_{0}$ & 61.8 & 2.6 & $61.7(61.3-65.0)$ & 62.0 & 2.7 & $61.8(61.3-65.0)$ & 0.388 \\
\hline & $\mathrm{T}_{1}$ & 61.5 & 3.0 & $61.6(61.3-61.8)$ & 61.6 & 3.3 & $61.6(61.3-65.0)$ & \\
\hline & $\mathrm{T}_{2}$ & 61.4 & 2.5 & $61.6(61.3-61.8)$ & 61.6 & 2.5 & $61.6(61.3-61.8)$ & \\
\hline \multirow[t]{3}{*}{$C^{*}{ }_{a b}$} & $\mathrm{~T}_{0}$ & 12.1 & 2.1 & $11.8(8.7-14.3)$ & 11.7 & 2.6 & $11.8(8.7-13.5)$ & 0.042 \\
\hline & $\mathrm{T}_{1}$ & 12.3 & 2.0 & $13.5(10.1-14.3)$ & 11.9 & 2.1 & $12.6(8.7-14.3)$ & \\
\hline & $\mathrm{T}_{2}$ & 12.7 & 1.7 & 13.5 (11.8-14.3) & 12.2 & 1.9 & $11.8(10.1-14.3)$ & \\
\hline \multirow[t]{3}{*}{$\Delta \mathrm{E}$} & $T_{0}-T_{1}$ & 2.10 & 2.57 & $0.90(0.00-4.22)$ & 2.46 & 2.82 & $1.88(0.00-4.42)$ & \\
\hline & $T_{1}-T_{2}$ & 2.15 & 2.54 & $1.88(0.00-4.36)$ & 2.35 & 2.50 & $1.88(0.00-4.36)$ & \\
\hline & $T_{0}-T_{2}$ & 1.80 & 2.21 & $0.90(0.00-3.48)$ & 1.86 & 2.17 & $1.01(0.00-3.60)$ & \\
\hline \multirow[t]{3}{*}{$\mathrm{d}(\mathrm{OM} 1)$} & $\mathrm{T}_{0}$ & 13.9 & 3.8 & $13.7(9.2-15.8)$ & 13.5 & 3.9 & $13.7(9.2-15.4)$ & 0.038 \\
\hline & $\mathrm{T}_{1}$ & 14.3 & 4.1 & $15.3(12.5-15.8)$ & 13.9 & 4.5 & $15.3(9.2-15.8)$ & \\
\hline & $T_{2}$ & 14.6 & 3.7 & $15.3(13.7-15.8)$ & 14.1 & 3.8 & 14.5 (12.5-15.8) & \\
\hline \multicolumn{9}{|l|}{$2 \mathrm{D}$ system ${ }^{c}$} \\
\hline \multirow[t]{3}{*}{$L^{*}$} & $\mathrm{~T}_{0}$ & 58.1 & 2.1 & $58.4(56.8-59.7)$ & 58.4 & 2.1 & $58.4(57.1-59.7)$ & 0.004 \\
\hline & $\mathrm{T}_{1}$ & 57.8 & 2.4 & $58.4(55.8-59.7)$ & 58.1 & 2.4 & $58.4(57.1-59.7)$ & \\
\hline & $\mathrm{T}_{2}$ & 57.7 & 2.3 & $57.1(55.8-59.7)$ & 58.1 & 2.0 & $58.4(57.1-59.7)$ & \\
\hline \multirow[t]{3}{*}{$C_{a b}^{*}$} & $\mathrm{~T}_{0}$ & 12.4 & 2.1 & $12.3(11.0-13.6)$ & 12.2 & 2.1 & $12.3(11.0-13.6)$ & 0.031 \\
\hline & $\mathrm{T}_{1}$ & 12.7 & 2.4 & $12.3(11.0-14.9)$ & 12.4 & 2.6 & $12.3(11.0-13.6)$ & \\
\hline & $\mathrm{T}_{2}$ & 12.9 & 2.0 & $13.6(11.0-14.9)$ & 12.5 & 2.3 & $12.3(11.0-13.6)$ & \\
\hline \multirow[t]{3}{*}{$\Delta \mathrm{E}$} & $T_{0}-T_{1}$ & 1.63 & 1.79 & $1.81(0.00-1.87)$ & 1.74 & 1.78 & $1.87(0.00-1.87)$ & \\
\hline & $T_{1}-T_{2}$ & 1.45 & 1.65 & $1.81(0.00-1.87)$ & 1.65 & 1.80 & $1.87(0.00-1.91)$ & \\
\hline & $T_{0}-T_{2}$ & 1.61 & 1.62 & $1.87(0.00-1.87)$ & 1.39 & 1.42 & $1.84(0.00-1.87)$ & \\
\hline
\end{tabular}

${ }^{3} 3 \mathrm{D}$ lightness values were assessed on a six-point integer scale from 0 to 5

$\mathrm{b}_{3 \mathrm{D}}$ chroma values were assessed on a three-point scale from 1 to 3 at half points

${ }^{\mathrm{C}} 2 \mathrm{D}$ second shade designation numbers were assessed on a five-point scale at quarter points

Gmd denotes Gini's mean difference (see statistical methods)

analysis syntax. Additionally to $L^{*}$, values for $a^{*}$ and $b^{*}$ were calculated from values of $C^{*} a b$ and $h$ degrees as given in Ahn et al. and were then used to calculate $\Delta E$ (defined [22] [as square root of $\left[\left(\Delta L^{*}\right)^{2}+\left(\Delta a^{*}\right)^{2}\right.$ $\left.\left.+\left(\Delta b^{*}\right)^{2}\right]\right)$. For example, the change from 1 M2 to 2 L2.5 was calculated in two steps. First, $a^{*}$ and $b^{*}$ values were calculated $\left(a^{*}{ }_{1 \mathrm{M} 2}=8.77^{*} \cos (89.4 * 2 * \pi / 360)\right.$ $\left.=0.09 ; b^{*}{ }_{1 \mathrm{M} 2}=8.7 * \sin (89.4 * 2 * \pi / 360)\right)$; then the square root of $\left[(65.0-61.3)^{2}+(0.09-0.82)^{2}+(8.70-13.5)^{2}\right]=6.1$ was calculated, which can also be found in Table III in $\mathrm{Ahn}$ et al. [21]. Because $\Delta E$ is restricted to non-negative values, we computed the distance of each shade to $0 \mathrm{M} 1$ additionally, denoted by $\mathrm{d}(0 \mathrm{M} 1)$. A positive change in $\mathrm{d}(0 \mathrm{M} 1)$ indicates a darker or stronger color; a negative change indicates a lighter or purer color.

In the $2 \mathrm{D}$-system, the shade group $\mathrm{B}$ is ordered by $C_{a b}^{*}$ (CIE chroma), but not by $L^{*}$ (CIE lightness); for the latter $\mathrm{B} 2>\mathrm{B} 1>\mathrm{B} 4>\mathrm{B} 3$ ) [16]. Therefore, we analyzed color distributions only in terms of $L^{*}, C^{*}{ }_{a b}$, and $\triangle \mathrm{E}$ after having assigned VITAPAN ${ }^{\circ}$ Classical shades to values given in the $\mathrm{D}_{65}$ columns of Table I in Park et al. [16] as described for the 3D system. Because the second shade designation numbers of the 
2D-system were assessed on a five-point scale at quarter points, extrapolation to five and interpolation to quarter points were applied.

In the 2D-system, the shade group B is ordered by $C^{*}{ }_{a b}$ (CIE chroma), but not by $L^{*}$ (CIE lightness); for the latter $\mathrm{B} 2>\mathrm{B} 1>\mathrm{B} 4>\mathrm{B} 3$ ) [16]. Therefore, we analyzed color distributions only in terms of $L^{*}, C^{*}{ }_{a b}$, and $\triangle \mathrm{E}$ after having assigned VITAPAN ${ }^{\circ}$ Classical shades to values given in the $\mathrm{D}_{65}$ columns of Table I in Park et al. [16] as described for the $3 \mathrm{D}$ system. Because the second shade designation numbers of the $2 \mathrm{D}$-system were assessed on a five-point scale at quarter points, extrapolation to five and interpolation to quarter points were applied.

As the American Statistical Association [23] recommends to avoid over-reliance on $p$-values, we estimated and interpreted confidence intervals [24]. Treatment effects were corrected for tooth level and subject level by using multilevel modeling [25], and adjusted for tooth type and quadrant. The group difference in change from baseline was calculated in order to estimate treatment effects. Originally, a difference in shade $\geq 3.7$ CIELAB units had been prespecified as clinically meaningful both for changes within groups and treatment effects [16] which was revised to $\geq 2.7$ [26]. The treatment group difference in change (change in $S_{1}$ versus change in $S_{2}$ ) was estimated by linear multilevel models with Kenward-Roger correction for small samples [27] via the procedure "mixed" by Stata software, release 14.2 (Stata Corporation, College Station, TX, USA); changes within groups were computed afterwards using the command "margin". The relative treatment effect of the difference in change was estimated by ordinal logistic multilevel models via Stata's procedure "meologit". Odds ratios in the ordinal logistic regression can be interpreted as those in the binary logistic regression whatever the cutoff point of the ordinal outcome is [28]. Box plots and descriptive statistics, including quantiles and Gini's mean difference (Gmd) as a robust measure of dispersion [28], were generated using R, release 3.3.3 (R Core Team (2017). R: A Language and Environment for Statistical Computing. R Foundation for Statistical Computing. Vienna, Austria. https:// www.r-project.org), especially the "ggplot2" package [29].

\section{Results}

\section{Subjects, teeth, and observations}

The initial study sample consisted of 26 consecutive patients. Eleven patients were excluded from the study for different reasons, including lack of oral hygiene, decalcification, or relocation. The multibracket appliances had been present in situ for 2.0 years $(S D \pm 0.3)$. At the end of MBA treatment, data for tooth color of 120 teeth of the upper jaw (\#14 to \#24) of 12 female and 3 males were available, resulting in a total of 720 observations for each color system (120 teeth, 2 tooth segments, 3 time points). All patients were Caucasian, aged 11 to 18 years.

\section{Measurements results 2D-system}

At baseline, 13 different shades were measured (Fig. 4a). Five shades with a frequency greater than 30 occurred: B2, B2.25, B2.5, B2.75 and B3 (Fig. 4a). Coordinates (CIE $\left.L^{*}, a^{*}, b^{*}\right)$ of quarter points for the second shade designation number were interpolated to $(61.0,59.7,58.4$, 57.1 , and 55.8) for $L^{*}$ of B2, B2.25, B2.5, B2.75 and B3, respectively, and to $(9.8,11.1,12.4,13.6$, and 14.9) for $C^{*}{ }_{a b}$ of B2, B2.25, B2.5, B2.75 and B3, respectively (Fig. 5). Note that B2.25, B2.5, and B2.75 lie in a space not well covered by the 3D-system (Fig. 5). Gingival segments were darker $\left(L^{*}\right)$ and stronger $\left(C^{*} a b\right)$ than body segments $(P=0.004$ and $P=0.031$, respectively; Table 1$)$.

Changes within segments $S_{1}$ and $S_{2}$ from baseline to 3 months after MBA treatment $\left(\mathrm{T}_{0}-\mathrm{T}_{2}\right)$ were at worst $1.97 \approx 2.0$ units $(\Delta \mathrm{E}$ for gingival segment; Table 2$)$, which is less than the threshold of 2.7 units for a clinical meaningful difference (Fig. 6a). Moreover, confidence intervals for the treatment effects in terms of the difference in change indicated no clinically important differences between body and gingival segments (Table 2).

\section{D-system}

At baseline, 13 different shades were measured (Fig. 4b). Four shades with a frequency greater than 30 occurred: 1 M2, 2 L2.5, 2 M3, and 3R2.5 (Fig. 4b). Note that shades $2 \mathrm{~L} 2.5,2 \mathrm{M} 3$, and 3R2.5 limit a space that is not well covered by the 3D system (Fig. 5; 3R2.5 is nearest neighbor of 3 L2.5). Chroma of gingival segments was stronger than that of body segments $(P=0.018$; Table 1$)$; differences in lightness were uncertain $(P=0.17$; Table 1$)$.

Changes within segments $S_{1}$ and $S_{2}$ from baseline to 3 months after MBA treatment $\left(\mathrm{T}_{0}-\mathrm{T}_{2}\right)$ were at worst $2.28 \approx 2.3$ units $(\Delta \mathrm{E}$ for body segment; Table 2$)$, which is less than the threshold of 2.7 units for a clinical meaningful difference. Figs. $6 \mathrm{~b}$ and 7 illustrate that $\Delta \mathrm{E}$ is prone to information bias (measurement error). The value of $\Delta \mathrm{E}=9.9$ for $\mathrm{T}_{0}-\mathrm{T}_{1}$ and $\mathrm{T}_{1}-\mathrm{T}_{2}$ at the gingival segment as shown in Fig. $6 \mathrm{~b}$ resulted from a change from $1 \mathrm{M} 2$ to $3 \mathrm{~L} 2.5$ and back to $1 \mathrm{M} 2$ for $\mathrm{T}_{0}, \mathrm{~T}_{1}$, and $\mathrm{T}_{2}$, respectively. This change is more appropriately described in terms of $\mathrm{d}(0 \mathrm{M} 1)$ : Values of $9.2,19.0$, and 9.2 for $\mathrm{T}_{0}, \mathrm{~T}_{1}$, and $\mathrm{T}_{2}$, respectively, correspond to a change in $\mathrm{d}(0 \mathrm{M} 1)$ of 9.8 , and -9.8 for $\mathrm{T}_{0}-\mathrm{T}_{1}$ and $\mathrm{T}_{1}-\mathrm{T}_{2}$, respectively, because $\mathrm{d}(0 \mathrm{M} 1)$ allows negative values to describe purer or lighter changes. Moreover, confidence intervals for the treatment effects in terms of the difference in change indicated no clinically important differences between body and gingival segments (Table 2). 

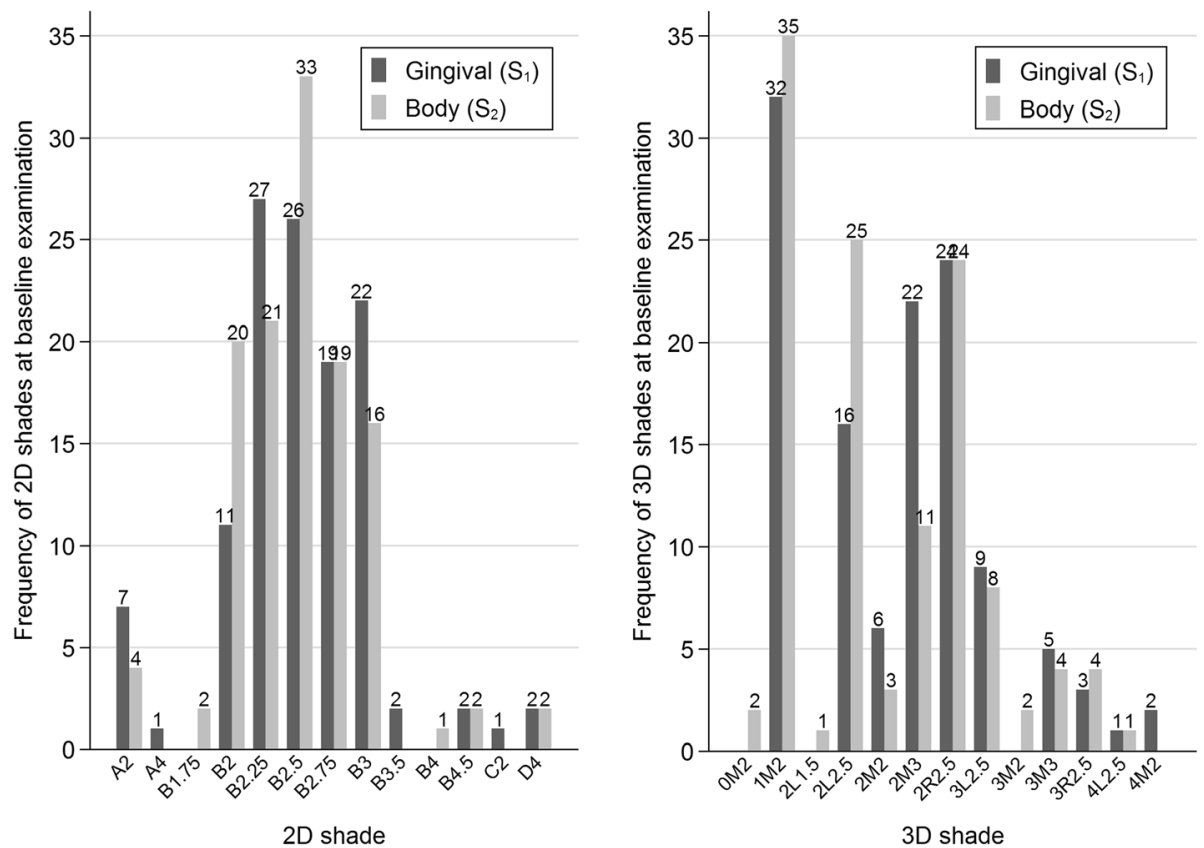

Fig. 4 a, b Frequencies of 2D and 3D shades in gingival and body segments of 120 teeth at baseline

\section{Discussion}

During MBA treatment, color changes in bracket (body) and non-bracket (gingival) tooth segments were not clinically relevant. Moreover, body and gingival tooth segments differed in change in tooth color only slightly and possibly by zero. The extent of change in color depended on color metrics (2D, 3D); nevertheless, our findings using different color metrics were sufficiently robust insofar as color change during MBA treatment was not clinically relevant, even if using small thresholds down to 2.3 units for a clinically relevant difference $(\Delta \mathrm{E})$.

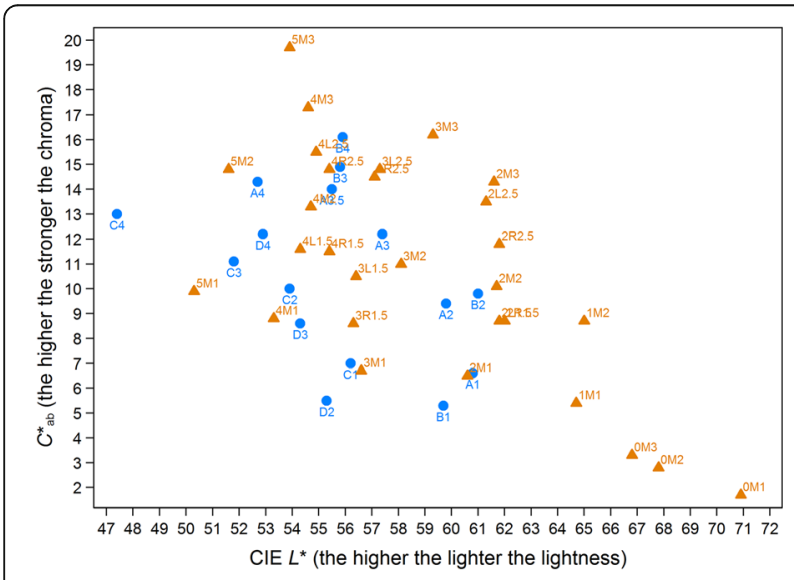

Fig. 5 Scatterplot of CIE $L^{*}$ and $C_{a b}^{*}$ values for $2 D$ shades (blue) and 3D shades (orange)

\section{Methods of the study}

In this study, we preferred electronical measurements instead of visual measurements for several reasons. First, it was assumed that problems due to the regression to the mean [30] which is "one of the most important of all phenomena regarding data and estimation" [31] could not have been substantially reduced by repeated visual measurements; the judger will be biased after the first measurement. Second, we aimed to use measurements of two systems (2D and 3D) for which judgers would have introduced bias regarding the second measurement. Third, four measurements as used internally by the electronic device to compute the overall value increased the reliability according to the Spearman-Brown formula. Fourth, by using quarter points, electronic 2D measurements could have been more accurate than visual 2D measurements. Finally, it could be expected that our adolescent patient group (11-18 years) was homogeneous concerning tooth colors, especially in terms of $\mathrm{B}$ color shades of the 2D system. Therefore, it could be assumed that a systematic measurement error will be substantially the same in this highly homogeneous group an assumption which would not be justified in a sample with a wide age range (and more frequent color shades different from $B$ of the 2D system). This is a crucial point because in presence of a constant systematic measurement error the validity of the measurement of change will not be threatened. In short, we looked for a trade-off between reliability and validity issues, including regression to the mean. 
Table 2 Treatment effects in terms of the difference in change using linear multilevel models to account for 15 subjects and 120 teeth, and relative treatment effects of the change in terms of the odds ratio of the body segment referred to the gingival segment using ordinal multilevel models

\begin{tabular}{|c|c|c|c|c|c|c|c|}
\hline & \multirow[b]{3}{*}{ Time points } & \multicolumn{4}{|c|}{ Linear multilevel model (mixed model) } & \multirow{2}{*}{\multicolumn{2}{|c|}{$\frac{\text { Ordinal multilevel model }}{\text { Relative treatment effect }}$}} \\
\hline & & \multirow{2}{*}{$\begin{array}{l}\text { Change within } \\
\text { gingival segment }\left(S_{1}\right) \\
(95 \% \mathrm{Cl})\end{array}$} & \multirow{2}{*}{$\begin{array}{l}\text { Change within } \\
\text { body segment }\left(\mathrm{S}_{2}\right) \\
\text { Change }(95 \% \mathrm{Cl})\end{array}$} & \multicolumn{2}{|l|}{$\begin{array}{l}\text { Treatment effect } \\
\text { (difference in change) }\end{array}$} & & \\
\hline & & & & Coefficient $(95 \% \mathrm{Cl})$ & $P$ value & Odds ratio $(95 \% \mathrm{Cl})$ & $P$ value \\
\hline \multicolumn{8}{|l|}{ 3D system } \\
\hline Lightness & $T_{0}-T_{1}$ & $0.09(-0.10-0.28)$ & $0.12(-0.07-0.31)$ & $0.025(-0.11-0.16)$ & 0.716 & $1.08(0.62-1.90)$ & 0.780 \\
\hline Lightness & $T_{1}-T_{2}$ & $0.04(-0.15-0.23)$ & $0.02(-0.17-0.22)$ & $-0.017(-0.15-0.12)$ & 0.807 & $0.95(0.54-1.66)$ & 0.860 \\
\hline Lightness & $T_{0}-T_{2}$ & $0.13(0.02-0.25)$ & $0.14(0.02-0.26)$ & $0.008(-0.11-0.12)$ & 0.885 & $1.05(0.57-1.94)$ & 0.870 \\
\hline Chroma & $T_{0}-T_{1}$ & $0.03(-0.05-0.11)$ & $0.01(-0.07-0.09)$ & $-0.017(-0.09-0.06)$ & 0.668 & $0.87(0.51-1.49)$ & 0.608 \\
\hline Chroma & $T_{1}-T_{2}$ & $0.07(-0.01-0.15)$ & $0.08(0.004-0.16)$ & $0.017(-0.06-0.09)$ & 0.648 & $1.16(0.68-1.98)$ & 0.587 \\
\hline Chroma & $T_{0}-T_{2}$ & $0.10(0.01-0.19)$ & $0.10(0.01-0.19)$ & $0.000(-0.7-0.07)$ & 1.000 & $0.97(0.56-1.69)$ & 0.911 \\
\hline$L^{*}$ & $\mathrm{~T}_{0}-\mathrm{T}_{1}$ & $0.34(-0.27-0.94)$ & $0.40(-0.20-1.00)$ & $0.066(-0.41-0.54)$ & 0.786 & $1.04(0.65-1.66)$ & 0.879 \\
\hline$L^{*}$ & $T_{1}-T_{2}$ & $0.03(-0.65-0.70)$ & $-0.004(-0.68-0.67)$ & $-0.033(-0.51-0.44)$ & 0.889 & $0.83(0.52-1.33)$ & 0.436 \\
\hline$L^{*}$ & $T_{0}-T_{2}$ & $0.37(-0.001-0.73)$ & $0.40(0.03-0.77)$ & $0.032(-0.37-0.43)$ & 0.873 & $0.92(0.57-1.47)$ & 0.726 \\
\hline$C_{a b}^{*}$ & $\mathrm{~T}_{0}-\mathrm{T}_{1}$ & $0.19(-0.43-0.81)$ & $0.18(-0.44-0.80)$ & $-0.008(-0.48-0.47)$ & 0.972 & $1.06(0.66-1.69)$ & 0.809 \\
\hline$C_{a b}^{*}$ & $\mathrm{~T}_{1}-\mathrm{T}_{2}$ & $0.42(-0.13-0.96)$ & $0.29(-0.26-0.83)$ & $-0.129(-0.55-0.29)$ & 0.543 & $0.85(0.53-1.36)$ & 0.498 \\
\hline$C_{a b}^{*}$ & $T_{0}-T_{2}$ & $0.61(0.19-1.03)$ & $0.47(0.05-0.89)$ & $-0.138(-0.51-0.23)$ & 0.464 & $0.78(0.49-1.25)$ & 0.302 \\
\hline$\Delta \mathrm{E}$ & $T_{0}-T_{1}$ & $2.10(1.50-2.69)$ & $2.46(1.86-3.05)$ & $0.360(-0.16-0.89)$ & 0.176 & $1.43(0.88-2.33)$ & 0.154 \\
\hline$\Delta \mathrm{E}$ & $T_{1}-T_{2}$ & $2.15(1.64-2.66)$ & $2.35(1.84-2.86)$ & $0.197(-0.33-0.72)$ & 0.460 & $1.23(0.77-1.97)$ & 0.392 \\
\hline$\Delta \mathrm{E}$ & $T_{0}-T_{2}$ & $1.80(1.39-2.22)$ & $1.86(1.45-2.28)$ & $0.062(-0.37-0.49)$ & 0.777 & $1.11(0.68-1.81)$ & 0.688 \\
\hline $\mathrm{d}(\mathrm{OM} 1)$ & $T_{0}-T_{1}$ & $0.38(-0.46-1.22)$ & $0.42(-0.41-1.26)$ & $0.043(-0.56-0.65)$ & 0.889 & $1.01(0.63-1.61)$ & 0.979 \\
\hline $\mathrm{d}(\mathrm{OM} 1)$ & $T_{1}-T_{2}$ & $0.32(-0.48-1.12)$ & $0.21(-0.59-1.01)$ & $-0.11(-0.66-0.44)$ & 0.691 & $0.92(0.58-1.47)$ & 0.739 \\
\hline $\mathrm{d}(\mathrm{OM} 1)$ & $T_{0}-T_{2}$ & $0.70(0.22-1.18)$ & $0.63(0.15-1.11)$ & $-0.068(-0.55-0.41)$ & 0.781 & $0.96(0.60-1.54)$ & 0.882 \\
\hline \multicolumn{8}{|l|}{ 2D system } \\
\hline$L^{*}$ & $\mathrm{~T}_{0}-\mathrm{T}_{1}$ & $0.31(-0.17-0.80)$ & $0.30(-0.19-0.78)$ & $-0.016(-0.35-0.32)$ & 0.926 & $1.01(0.63-1.60)$ & 0.975 \\
\hline$L^{*}$ & $T_{1}-T_{2}$ & $0.06(-0.37-0.50)$ & $0.05(-0.39-0.49)$ & $-0.012(-0.31-0.29)$ & 0.936 & $0.91(0.57-1.46)$ & 0.704 \\
\hline$L^{*}$ & $T_{0}-T_{2}$ & $0.38(-0.03-0.78)$ & $0.33(-0.06-0.75)$ & $-0.028(-0.31-0.26)$ & 0.847 & $0.93(0.58-1.48)$ & 0.748 \\
\hline$C_{a b}^{*}$ & $\mathrm{~T}_{0}-\mathrm{T}_{1}$ & $0.23(-0.29-0.75)$ & $0.17(-0.35-0.69)$ & $-0.053(-0.36-0.26)$ & 0.738 & $0.84(0.53-1.33)$ & 0.449 \\
\hline$C_{a b}^{*}$ & $\mathrm{~T}_{1}-\mathrm{T}_{2}$ & $0.20(-0.25-0.66)$ & $0.17(-0.28-0.62)$ & $-0.034(-0.31-0.24)$ & 0.805 & $0.90(0.56-1.44)$ & 0.668 \\
\hline$C_{a b}^{*}$ & $T_{0}-T_{2}$ & $0.43(0.05-0.82)$ & $0.34(-0.04-0.73)$ & $-0.087(-0.33-0.15)$ & 0.471 & $0.77(0.49-1.23)$ & 0.279 \\
\hline$\Delta \mathrm{E}$ & $T_{0}-T_{1}$ & $1.63(1.17-2.10)$ & $1.74(1.28-2.21)$ & $0.111(-0.26-0.48)$ & 0.552 & $1.33(0.83-2.14)$ & 0.239 \\
\hline$\Delta \mathrm{E}$ & $\mathrm{T}_{1}-\mathrm{T}_{2}$ & $1.45(1.03-1.87)$ & $1.65(1.22-2.07)$ & $0.199(-0.16-0.56)$ & 0.271 & $1.32(0.82-2.13)$ & 0.260 \\
\hline$\Delta \mathrm{E}$ & $T_{0}-T_{2}$ & $1.61(1.24-1.97)$ & $1.39(1.03-1.76)$ & $-0.214(-0.51-0.08)$ & 0.155 & $0.80(0.49-1.28)$ & 0.346 \\
\hline
\end{tabular}

Nevertheless, there are some limitations concerning the electronical measurement methods, including light condition, calibration of the measurement device, reproducibility of the measurements, and visual threshold discussed in the literature [32]. The spectrophotometer Shade Inspector $^{\text {ru }}$ was used in our study, because of its good results regarding reproducibility of lightness and chroma found in pilot studies $[15,33]$. Other studies, investigating dental color measuring devices did show reliable results as well [34-38].
The Shade Inspector ${ }^{\mathrm{rm}}$ is calibrated with a factory-provided selection of industrially fabricated color reference scale (VITAPAN ${ }^{\circ}$ Classical and VITA $3 D$-Master ${ }^{\circ}$. These color scales originating of different batches were read in and the measurements averaged. Therefore, variations in measurements due to the calibration process are conceivably [39]. The study of Kohlmeyer and Scheller evaluating VITAPAN ${ }^{\circ}$ Classical color scale samples, revealed that the individual color scale samples failed to invariably correspond to the respective primary color [40]. In addition, unequivocal findings 

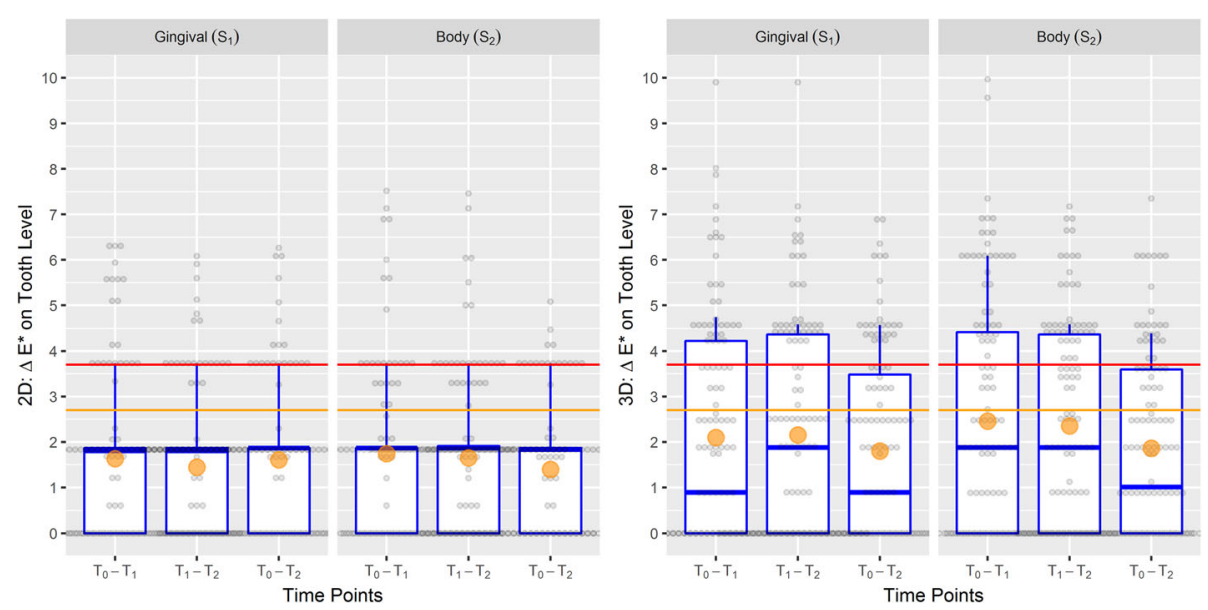

Fig. $\mathbf{6}$ a , b Box plots showing the distribution of $\Delta \mathrm{E}$ for the 2D-system (a; left) and the 3D-system (b; right) on tooth level. Orange circle: mean; bold line: median; box: interquartile range (between 25 and 75\%); whiskers: range between 12.5 and 87.5\%; grey dots figure the 120 observations; red line: clinically important difference at 3.7 units or 2.7 units

were reported on color consistency alongst shade guides from the same manufacturer $[41,42]$. One in vitro study found that repeatability and accuracy of a dental color measuring instrument (ShadeScan) was influenced by shade guide systems used for testing [43]. In our study, the complete clinical procedure was performed by an experienced orthodontist under standardized conditions (color neutral such as same room, same dental unit and same light conditions by dental unit lamp, patient was covered by a drape, tooth surfaces were always saliva-wet). The electronical measurements were performed by a calibrated examiner [15] in a pilot study. The tooth color measuring device itself operates independently of light on the principle of spectral photometry. However, in a study, evaluating the effect of different illuminants (natural daylight, dental unit lamp, and daylight lamp), the matching repeatability of 2 intraoral spectrophotometers was not completely satisfactory for clinical practice [44]. Therefore, our measurements were taken under standardized conditions as described before. Thus, we do not assume relevant effects by the surrounding light conditions.

Our study has methodological strengths. Notably, two measurements $(2 \mathrm{D}, 3 \mathrm{D})$ at each time point were used, thereby reducing problems due to regression to the mean, which is here the tendency of tooth segment's colors at the extremes to have less extreme values on subsequent measurements [30]. To reduce the influence of extreme values at the first measurement, it is common to discard the first of three blood pressure measurements of the same examination [45] or to measure the periodontium by the Florida probe thrice given disagreement in first two measurements. Importantly for interpreting of the analysis of change as done herein, the second measurement was performed by the 3D-system, which was considered as the primary outcome. Moreover, we used mixed models as a shrinkage approach and "a way of discounting observed variation that accounts for regression to the mean" [31]. Second, the 2D-system measured at quarter points for the second shade designation number. As the 3D-system did not cover the space of the most frequent $2 \mathrm{D}$ shades, the 2D-system added essential information, although limited by the regression to the mean. Third, tooth type as a potentially substantial confounder can only be considered in multilevel analysis. Further, it is not possible to address confounding due to tooth type by the study design. Thus, tooth type cannot be subject of randomizing in a MB study; analysis restricted to the subject level can be misleading. Fourth, we presented not only the original codes of the 2D- and 3D-system but also the transformed values based on the CIE system. As $\mathrm{B} 2>\mathrm{B} 1>$ B4 > B3 on the $L^{*}$ scale [16]. the shade designation numbers of the original 2D codes cannot be well interpreted. Finally, we used not only $\Delta \mathrm{E}$ to estimate the treatment effect but also the measure $\mathrm{d}(0 \mathrm{M} 1)$ to allow for purer or lighter changes. In terms of $L^{*}, \Delta \mathrm{E}$ does not differentiate a lighter change from a darker change given the same $\Delta \mathrm{E}$; in terms of $C^{*}{ }_{a b}, \Delta \mathrm{E}$ does not differentiate a purer change from a stronger change. The $3 \mathrm{D}$ shade $0 \mathrm{M} 1$ as the new origin of the coordinate system enables us to differentiate lighter/purer changes from darker/ stronger changes. $0 \mathrm{M} 1$ as the new origin of the 3D-system is justified for its lightest lightness and its purest chroma, including the purest red $\left(a^{*}\right)$ and the purest yellow $\left(b^{*}\right)$. For the 2D- system, no shade has these properties [16].

Unfortunately, there was no sample size calculation for this study. However, we accounted for subject and tooth level to increase statistical power. Moreover, other 

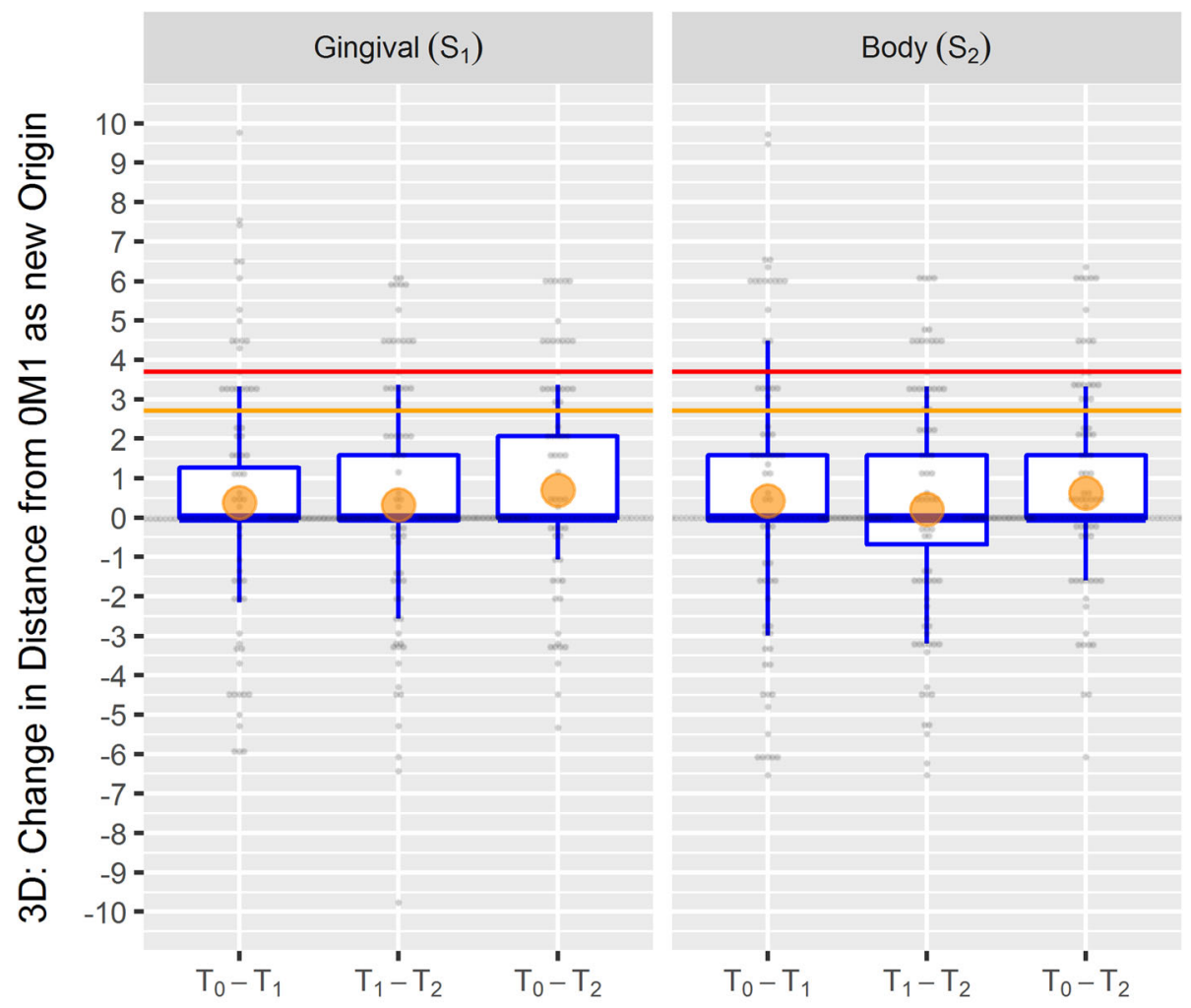

Time Points

Fig. 7 Box plots showing the distribution of the change in distance from $0 \mathrm{M} 1$ for the 3D system on tooth level. Orange circle: mean; bold line: median; box: interquartile range (between 25 and 75 - 50\% of the values); whiskers: range between the 12.5 and $87.5 \%$ (75\% of the values); grey dots figure the 120 observations; change $>0$ indicates darker or stronger colors; change $<0$ indicates lighter or purer colors; red line: clinically important difference at 3.7 units or 2.7 units

studies included similar numbers of participants $[10,46]$. Besides this limitation, it was not sensitive to adjust for baseline values [47-49], because segments could not be randomized to treatment groups. Therefore, we compared the difference in change from baseline between segments $[28,50,51]$.

\section{Discussion of results}

Confidence intervals for the treatment effect for both color systems indicated no clinically important differences between body and gingival segments. Further, changes from baseline to 3 months after MBA treatment $\left(\mathrm{T}_{0}-\mathrm{T}_{2}\right)$ were at worst 2.3 units for $3 \mathrm{D}$ - system and 2.0 units for $2 \mathrm{D}$-system, respectively, which are less than the threshold of 2.7 units for a clinical meaningful difference.

Previous studies $[4,14]$ have shown that the enamel color variables are affected by orthodontic bonding and debonding procedures due to tooth cleaning [52], enamel conditioning procedures (etching) [53], and enamel scratches [54]. Other effects, such as staining of enamel and resin material used for the bonding brackets, may also induce color change of teeth during orthodontic treatment. These color change may be the result of demineralization [55], or direct food dye $[12,56]$. The staining of the resin material is associated with the color instability of the polymer [57].

Several experimental studies $[3,4,12-14,58]$ investigated the impact of the bonding process on tooth color. Three studies $[3,12,14]$ investigating color change after bonding of extracted teeth have not found any indication of a significant influence of the bonding process on tooth color. In another experimental study [13] assessing color changes in bracket areas, significant differences in $\Delta \mathrm{E}$ were found. Despite the significance of the results, the authors did not consider the color changes visually perceivable for the majority of examiners. Eliades et al. [4] reached similar conclusions when examining the influence of different bonding materials. Furthermore, enamel color alterations might also derive from the irreversible penetration of resin into the enamel surface [4]. Moderate evidence exits that shorter resin tags penetration produces less change in enamel color following clean-up procedure and polishing [58]. Self-etching 
primers produce less resin penetration and these systems produce less iatrogenic color change in enamel following orthodontic treatment [58]. In our study 35\%-phosphoric acid was used.

The results of a prospective clinical trial conducted by Karamouzos et al. [10] showed significant changes of tooth color (2.1 to $3.6 \Delta \mathrm{E}$ units) after orthodontic treatment. The value for the parameter lightness $\left(\mathrm{L}^{*}\right)$ decreased, whereas the values for the parameters $\mathrm{a}^{*}$ (value for green-red) and $b^{*}$ (value for blue-yellow) increased. These changes indicated a decrease in tooth lightness as well as a change in hue, which may be perceptible if a threshold of 1.2 is assumed [26]. In our study, however, we did not find $\Delta \mathrm{E}$ values greater than 2.7 units, which are considered clinically relevant [26]. Nevertheless, our results are in accordance to a recently published review by Chen that there is no strong evidence that orthodontic treatment with fixed appliances alters the original color of enamel [8].

\section{Conclusion}

Within the limitation of this study the MBA treatment can be seen as a safe method with respect to tooth color.

\section{Funding}

The study was not funded.

\section{Availability of data and materials}

All data are available on request at Department of Orthodontics. Dental school, University Medicine, Walther-Rathenau Straße 42a, 17,475 Greifswald, Germany.

\section{Informed consent}

Informed consent was obtained from all individual participants and parents included in the study.

\section{Explanation of any issues relating to journal policies} No issues.

\section{Authors' contributions}

AR Contributed to data acquisition, design, analysis and interpretation, writing of manuscript. CS Contributed to statistical analysis and interpretation, writing statistical part of manuscript. AT Contributed to data acquisition. AF Critically revised the manuscript. AW Contributed to design, analysis and interpretation, writing of manuscript. All authors gave final approval and agree to be accountable for all aspects of the work. All authors have read and approved the manuscript.

\section{Ethics approval and consent to participate}

All procedures performed in this study involving human participants were in accordance with the ethical standards of the institutional research committee Ärztekammer Mecklenburg-Vorpommern (Reg. Nr.III UV 15/08) and with the 1964 Helsinki declaration and its later amendments or comparable ethical standards.

\section{Consent for publication}

The authors confirm that the content of the manuscript has not been published or submitted for publication elsewhere.

\section{Competing interests}

The authors declare that they have no competing interests.

\section{Publisher's Note}

Springer Nature remains neutral with regard to jurisdictional claims in published maps and institutional affiliations.

\section{Author details}

${ }^{1}$ Department of Orthodontics and Department of Dental Propaedeutics/ Community Dentistry, Dental School, University Medicine, Walther-Rathenau Straße 42a, 17475 Greifswald, Germany. ${ }^{2}$ Department of Prosthetic Dentistry, Gerodontology and Biomaterials, University of Greifswald, Fleischmannstraße 42, 17475 Greifswald, Germany. ${ }^{3}$ Private Dental Office, Bahnhofstraße 4, 18581 Putbus, Germany. ${ }^{4}$ Department of Orthodontics, Dental School, University Medicine, Franz-Josef-Strauß-Allee 11, 93053 Regensburg, Germany. ${ }^{5}$ Department of Restorative Dentistry, Periodontology, Endodontology, Preventive and Pediatric Dentistry, Dental School, University Medicine, Walther-Rathenau Straße 42a, 17475 Greifswald, Germany.

Received: 11 December 2017 Accepted: 17 September 2018

Published online: 22 October 2018

\section{References}

1. Watts A, Addy M. Tooth discolouration and staining: a review of the literature. Br Dent J. 2001;190:309-16. https://doi.org/10.1038/sj.bdj. 4800959a

2. Burrows S. A review of the safety of tooth bleaching. SADJ. 2010;65(6):8-13.

3. Wriedt $\mathrm{S}$, Keller $\mathrm{S}$, Wehrbein $\mathrm{H}$. The effect of debonding and/or bleaching on enamel color - an in-vitro study. J Orofac Orthop. 2008:69:169-76. https://doi.org/10.1007/s00056-008-0737-2.

4. Eliades T, Kakaboura A, Eliades G, Bradley TG. Comparison of enamel colour changes associated with orthodontic bonding using two different adhesives. Eur J Orthod. 2001;23:85-90.

5. Silverstone LM, Saxton CA, Dogon IL, Fejerskov O. Variation in the pattern of acid etching of human dental enamel examined by scanning electron microscopy. Caries Res. 1975;9:373-87.

6. Baumann DF, Brauchli L, van Waes $H$. The influence of dental loupes on the quality of adhesive removal in orthodontic debonding. J Orofac Orthop. 2011;72:125-32. https://doi.org/10.1007/s00056-011-0010-y.

7. Ogaard B, Fjeld M. The enamel surface and bonding in orthodontics. Semin Orthod. 2010;16:37-48

8. Chen Q, Zheng X, Chen W, Ni Z, Zhou Y. Influence of orthodontic treatment with fixed appliances on enamel color: a systematic review. BMC Oral Health. 2015:15:31. https://doi.org/10.1186/s12903-015-0014-x.

9. Janiszewska-Olszowska J, Szatkiewicz T, Tomkowski R, Tandecka K, Grocholewicz K. Effect of orthodontic debonding and adhesive removal on the enamel - current knowledge and future perspectives - a systematic review. Med Sci Monit. 2014;20:1991-2001. https://doi.org/10.12659/MSM. 890912

10. Karamouzos A, Athanasiou AE, Papadopoulos MA, Kolokithas G. Tooth-color assessment after orthodontic treatment: a prospective clinical trial. Am J Orthod Dentofac Orthop. 2010;138(537):e531-8; discussion 537-539. https:// doi.org/10.1016/j.ajodo.2010.03.026.

11. Boncuk Y, Cehreli ZC, Polat-Ozsoy O. Effects of different orthodontic adhesives and resin removal techniques on enamel color alteration. Angle Orthod. 2014;84:634-41. https://doi.org/10.2319/060613-433.1.

12. Trakyali G, Ozdemir Fl, Arun T. Enamel colour changes at debonding and after finishing procedures using five different adhesives. Eur J Orthod. 2009; 31:397-401. https://doi.org/10.1093/ejo/cjp023.

13. Jahanbin A, Ameri $H$, Khaleghimoghaddam R. Effect of adhesive types on enamel discolouration around orthodontic brackets. Aust Orthod J. 2009;25:19-23.

14. Hintz JK, Bradley TG, Eliades T. Enamel colour changes following whitening with 10 per cent carbamide peroxide: a comparison of orthodonticallybonded/debonded and untreated teeth. Eur J Orthod. 2001;23:411-5.

15. Ratzmann A, Klinke T, Schwahn C, Treichel A, Gedrange T. Reproducibility of electronic tooth colour measurements. Biomed Tech (Berl). 2008;53:259-63. https://doi.org/10.1515/BMT.2008.036.

16. Park JH, Lee YK, Lim BS. Influence of illuminants on the color distribution of shade guides. J Prosthet Dent. 2006;96:402-11. https://doi.org/10.1016/j. prosdent.2006.10.007.

17. Paravina RD. Performance assessment of dental shade guides. J Dent. 2009; 37(Suppl 1):e15-20. https://doi.org/10.1016/j.jdent.2009.02.005.

18. Vita Z. Dental Shade Guides. In J Am Dent Assc. 2002;133:366-7. 
19. Cook RJ, Farewell VT. Multiplicity considerations in the design and analysis of clinical trials. J R Stat Soc Ser. 1996;159:93-110. https://doi.org/10.2307/ 2983471.

20. $\quad$ ICH E9 Expert Working Group. Statistical principles for clinical trials: ICH harmonized tripartite guideline. Stat Med. 1999;18:1905-42.

21. Ahn JS, Lee YK. Color distribution of a shade guide in the value, chroma, and hue scale. J Prosthet Dent. 2008;100:18-28. https://doi.org/10.1016/ S0022-3913(08)60129-8

22. Paravina RD, Powers JM, Fay RM. Color comparison of two shade guides. Int J Prosthodont. 2002;15:73-8.

23. Wasserstein RL, Assoc AS. ASA statement on statistical significance and P-values. Am Stat. 2016;70:131-3. https://doi.org/10.1080/00031305.2016 1154108.

24. Greenland S, Senn SJ, Rothman KJ, Carlin JB, Poole C, Goodman SN, Altman DG. Statistical tests, $P$ values, confidence intervals, and power: a guide to misinterpretations. Eur J Epidemiol. 2016;31:337-50. https://doi. org/10.1007/s10654-016-0149-3.

25. Rabe-Hesketh S, Skrondal A. Multilevel and longitudinal modeling using Stata. 3rd ed. College Station: Stata Press; 2012

26. Paravina RD, Ghinea $R$, Herrera $L J$, Bona AD, Igiel C, Linninger $M$, Sakai $M$, Takahashi H, Tashkandi E, Perez MM. Color difference thresholds in dentistry. J Esthet Restor Dent. 2015;27(Suppl 1):S1-9. https://doi.org/10.1111/jerd. 12149.

27. Kenward MG, Roger JH. Small sample inference for fixed effects from restricted maximum likelihood. Biometrics. 1997;53:983-97.

28. Harrell FE Jr. With applications to linear models, logistic and ordinal regression, and survival analysis. In: Regression modeling strategies. 2nd ed. Heidelberg: Springer; 2015. https://doi.org/10.1007/978-3-31919425-7.

29. Wickham H. Elegant graphics for data analysis. New York: Springer; 2009

30. Fletcher RH, Fletcher SW, Fletcher GS. Clinical epidemiology: the essentials. 5th ed. Philadelphia: Lippincott Williams \& Wilkins; 2014

31. Harrell FE, Jr., Slaughter JE. Biostatistics for biomedical research. Harell FE ed. pp. 1- 11-22-11: biostat.mc.vanderbilt.edu; 2017. http://www.fharrell.com/. Accessed 25 May 2018

32. Chu SJ, Trushkowsky RD, Paravina RD. Dental color matching instruments and systems. Review of clinical and research aspects. J Dent 2010;38 Suppl 2:e2-16; doi: https://doi.org/10.1016/j.jdent.2010.07.001

33. Ratzmann A, Treichel A, Langforth G, Gedrange T, Welk A. Experimental investigations into visual and electronic tooth color measurement. Biomed Tech (Berl). 2011;56:115-22. https://doi.org/10.1515/BMT.2011.008.

34. Ishikawa-Nagai S, Ishibashi K, Tsuruta O, Weber HP. Reproducibility of tooth color gradation using a computer color-matching technique applied to ceramic restorations. J Prosthet Dent. 2005;93:129-37.

35. Da Silva JD, Park SE, Weber HP, Ishikawa-Nagai S. Clinical performance of a newly developed spectrophotometric system on tooth color reproduction. J Prosthet Dent. 2008;99:361-8. https://doi.org/10.1016/ S0022-3913(08)60083-9.

36. Dozic A, Kleverlaan CJ, El-Zohairy A, Feilzer AJ, Khashayar G. Performance of five commercially available tooth color-measuring devices. J Prosthodont. 2007;16:93-100. https://doi.org/10.1111/j.1532-849X.2007.00163.x.

37. Kim-Pusateri S, Brewer JD, Davis EL, Wee AG. Reliability and accuracy of four dental shade-matching devices. J Prosthet Dent. 2009;101:193-9. https://doi. org/10.1016/S0022-3913(09)60028-7.

38. Olms C, Setz JM. The repeatability of digital shade measurement--a clinical study. Clin Oral Investig. 2013;17:1161-6. https://doi.org/10.1007/ s00784-012-0796-z.

39. Hugo B, Witzel T, Klaiber B. Comparison of in vivo visual and computeraided tooth shade determination. Clin Oral Investig. 2005;9:244-50. https:// doi.org/10.1007/s00784-005-0014-3

40. Kohlmeyer B, Scheller H. Computerised tooth color determination with measuring device "digital shade guide". Dtsch Zahnarztl Z. 2002;57:172-5.

41. Cal E, Sonugelen M, Guneri P, Kesercioglu A, Kose T. Application of a digital technique in evaluating the reliability of shade guides. J Oral Rehabil. 2004; 31:483-91.

42. Tashkandi E. Consistency in color parameters of a commonly used shade guide. Saudi Dent J. 2010;22:7-11. https://doi.org/10.1016/j.sdentj. 2009.12.002

43. Kim-Pusateri S, Brewer JD, Dunford RG, Wee AG. In vitro model to evaluate reliability and accuracy of a dental shade-matching instrument. J Prosthet Dent. 2007;98:353-8. https://doi.org/10.1016/S0022-3913(07)60119-X.
44. Sarafianou A, Kamposiora P, Papavasiliou G, Goula H. Matching repeatability and interdevice agreement of 2 intraoral spectrophotometers. J Prosthet Dent. 2012;107:178-85. https://doi.org/10.1016/S0022-3913(12)60053-5.

45. Friedman LM, Furberg CD, DL DM, Reboussin DM, granger CB. Fundamentals of Clinical Trials. 5th ed. Heidelberg: Springer; 2015.

46. Hammad SM, El Banna M, El Zayat I, Mohsen MA. Effect of resin infiltration on white spot lesions after debonding orthodontic brackets. Am J Dent. 2012;25:3-8.

47. Cologne JB. Re: "when is baseline adjustment useful in analyses of change? An example with education and cognitive change". Am J Epidemiol. 2006; 164:1138-9. https://doi.org/10.1093/aje/kwj359.

48. Chen JT, Weuve J, Glymour M, Rehkopf D. Quantifying survivor bias in lifecourse epidemiologic studies. Am J Epidemiol. 2006;163:S69.

49. Glymour MM, Weuve J, Berkman LF, Kawachi I, Robins JM. When is baseline adjustment useful in analyses of change? An example with education and cognitive change. Am J Epidemiol. 2005;162:267-78. https://doi.org/10.1093/Aje/Kwi187.

50. Senn S. Change from baseline and analysis of covariance revisited. Stat Med. 2006;25:4334-44. https://doi.org/10.1002/sim.2682.

51. Harrell FE, Jr.: Statistical Errors in the Medical Literature. 2017; http://www. fharrell.com/post/errmed/\#change. Accessed 16 April 2018.

52. Thompson RE, Way DC. Enamel loss due to prophylaxis and multiple bonding/debonding of orthodontic attachments. Am J Orthod. 1981;79: 282-95.

53. van Waes H, Matter T, Krejci I. Three-dimensional measurement of enamel loss caused by bonding and debonding of orthodontic brackets. Am J Orthod Dentofac Orthop. 1997;112:666-9.

54. Sandison RM. Tooth surface appearance after debonding. Br J Orthod. 1981; 8:199-201.

55. Ogaard B, Rolla G, Arends J. Orthodontic appliances and enamel demineralization. Part 1. Lesion development. Am J Orthod Dentofac Orthop. 1988;94:68-73

56. Eliades T, Gioka C, Heim M, Eliades G, Makou M. Color stability of orthodontic adhesive resins. Angle Orthod. 2004;74:391-3. https://doi.org/ 10.1043/0003-3219(2004)074<0391:CSOOAR>2.0.CO;2.

57. Faltermeier A, Rosentritt M, Reicheneder C, Behr M. Discolouration of orthodontic adhesives caused by food dyes and ultraviolet light. Eur J Orthod. 2008;30:89-93. https://doi.org/10.1093/ejo/cjm058.

58. Zaher AR, Abdalla EM, Abdel Motie MA, Rehman NA, Kassem H, Athanasiou AE. Enamel colour changes after debonding using various bonding systems. J Orthod. 2012;39:82-8. https://doi.org/10.1179/1465312512Z.0000000009.

Ready to submit your research? Choose BMC and benefit from:

- fast, convenient online submission

- thorough peer review by experienced researchers in your field

- rapid publication on acceptance

- support for research data, including large and complex data types

- gold Open Access which fosters wider collaboration and increased citations

- maximum visibility for your research: over $100 \mathrm{M}$ website views per year

At $\mathrm{BMC}$, research is always in progress.

Learn more biomedcentral.com/submissions 